The design of the experiment shows up a second feature of the action of X-rays ; namely, that of chromosome stickiness. In the above combined experiments, the condition was non-existent in all the fixations made. It thus seems confirmed that stickiness owes its causation to an effect on the prophase; possibly, as we have suggested, by the superimposing of unpolymerized deoxyribose nucleic acid on the chromosome thread at this stage-probably early prophase, since sister reunion of unbroken ends coincides with stickiness ${ }^{1}$.

Finally, there was no breakage in the deoxyribose nucleic acid - starved heterochromatin following irradiation with X-rays of what must have been meta. phase chromosomes. This would seem to indicate that it is not deoxyribose nucleic acid which prevents the breakage of the charged heterochromatin in the resting stage, or prevents the direct breakage of charged metaphase chromosomes.

$$
\text { L. F. LA Cour }
$$

John Innes Horticultural Institution,

Bayfordbury, Hertford, Herts.

$$
\text { Nov. } 29 .
$$

${ }^{1}$ Darlington, C. D., and La Cour, I. F., J. Genet., 46, 180 (1945). 'Darlinaton. C. D.. and La Cour. L. F., J. Genet., 40, 185 (1940).

\section{Penetration of the Insect Cuticle by Isomers of Benzene Hexachloride}

IN the course of work on the penetration of benzene hexachloride through the insect integument, grain weevils (Sitophilus granarius) have been exposed to filter papers impregnated with pure isomers of benzene hexachloride, and the amount taken up by the weevils determined by a micro-colorimetric method capable of estimating amounts of $10-500 \mu \mathrm{gm}$.

The benzene hexachloride was determined as two fractions, an 'outside' fraction obtained by washing the insects with cold methanol, and an 'inside' fraction obtained by decomposing the methanolwashed insects with nitric acid and steam distilling out the benzene hexachloride so liberated.

It was expected that the methanol washing would remove superficial benzene hexachloride from insects, and that the 'outside' figures for recovered benzene hexachloride would be much the same for the four isomers, $\alpha, \beta, \gamma$ and $\delta$. The results given in the accompanying table show that this is not the case. The amounts of the four isomers recovered by methanol washings follow closely the order of their solubilities in certain solvents, notably hydrocarbons, esters and ether. It appears probable, therefore, that the initial stage in the pick-up of insecticide by the insect was solution in an outer layer, possibly the outer waxy layer of the epicuticle. Wigglesworth ${ }^{1}$ and Beament ${ }^{2}$ have described this waxy layer, and Beament has given a method for estimating its amount. We have estimated the amount of the waxy layer in the grain weevil, following closely the technique described by Beament, and find a figure of $6.9 \times 10^{-4} \mathrm{gm}$. per gm. of grain weevils. In the table, the maximum amounts of each isomer found 'outside' (after 7-12 hr. exposure) are expressed as 'solubilities' in gm. per $100 \mathrm{gm}$. on the assumption that they are dissolved in the amount of wax found. It will be seen that the figures bear a striking similarity to the solubilities of the isomers in a Grade 5 petroleum. This result is of interest in connexion with the findings of Bergmann ${ }^{8}$ and others that the
Penetration of Isomers of Brinzene Hexadhloride into Grain

\begin{tabular}{|c|c|c|c|c|}
\hline $\begin{array}{c}\text { Amount ( } \mu \mathrm{gm} \text {.) found per gm. insect } \\
\text { after 7-12 hr. exposure to fllter paper } \\
\text { impregnated at 11 } \mu \mathrm{gma} . / \mathrm{cm} .\end{array}$ & \multicolumn{4}{|c|}{ Isomer } \\
\cline { 2 - 5 } & $\alpha$ & $\beta$ & $\gamma$ & $\delta$ \\
\hline $\begin{array}{c}\text { 'Outside' (cold methanol wash) } \\
\text { 'Inside' (decomposed by nitric acid } \\
\text { and steam distilled) }\end{array}$ & 12 & 3 & 60 & 102 \\
$\begin{array}{c}\text { 'Solubility' of isomer in wax of epi- } \\
\text { cuticle (gm./100 gm. solvent) } \\
\text { Solubility in high-boiling petroleum } \\
\text { oil (B.R. 90\% }>320^{\circ} \text { C.) (gm./100 } \\
\text { gm. solvent) }\end{array}$ & $1 \cdot 7$ & 4 & 43 & 8 \\
\hline
\end{tabular}

wax layer of the epicuticle is a mixture of paraffins and long-chain esters.

Another interesting outcome of the work is that the 'inside' determinations show much greater penetration by $\gamma$-benzene hexachloride than by any of the other three isomers during the first twelve hours of exposure (see table). This is at variance with the conclusions, drawn by Dresden and Krijgsman ${ }^{4}$ from their injection experiments, that structural differences between the isomers are concerned only with toxicity at the site of action and do not affect penetration.

We are indebted to Mr. H. K. Southern and Mr. A. Williams for the micro-colorimetric method which formed the basis of this work.

A full account is being published elsewhere.

$$
\begin{aligned}
& \text { G. ARMSTRONG } \\
& \text { F. R. BRADBURy }
\end{aligned}
$$

Research Department,

Imperial Chemical Industries, Ltd.,

General Chemicals Division, Widnes. Oct. 19.

1 Wigglesworth, V. B., J. Exp. Biol., 21, 97 (1945).

a Beament, J. W. L., J. Exp. Biol., 21, 115 (1945).

${ }^{3}$ Bergmann, w., Ent. Soc. Amer., 31, 315 (1938).

4 Dresden, O., and Krijgsman, B. J., Bull. Ent. Res., 38, 575 (1948).

\section{Toxicity of Penicillin to Guinea Pigs}

IT has been reported ${ }^{1}$ that guinea pigs dosed with penicillin were more susceptible to experimental tuberculosis than were undosed controls. It has since been shown that penicillin per se is toxic to guinea pigs, and that this accounted for the earlier deaths in groups treated with penicillin ${ }^{2}$.

The work now being reported confirms the toxicity of amorphous coloured penicillin. A daily intramuscular dose of 20,000 units in aqueous solution for ten days resulted in the death of thirteen out of twenty guinea pigs in three to ten days. Pure crystalline penicillin given in the same dose and for the same duration resulted in the death of fifteen out of twenty.

It has further shown that both amorphous coloured and crystalline penicillin, in the same dose and duration as above, but previously inactivated by penicillinase, caused no deaths in forty treated guinea pigs. A single dose of 20,000 units of amorphous coloured penicillin given at the same time as 2 c.c. of penicillinase intramuscularly in different thighs caused six deaths in ten animals so treated.

Smaller doses, for example, a single dose of 20,000 units of crystalline penicillin, resulted in the death of nine out of twenty guinea pigs in from three to six days, and five daily doses of 1,000 units of amorphous coloured penicillin killed six out of twenty guinea pigs in from three to six days.

There were no deaths in control undosed animals over the period of observation. 